\title{
Interaction of Human Serum Albumin and Silica at the air-water interface
}

\author{
Joo Chuan Ang ${ }^{\mathrm{a}}$, Jhih-Min Lin ${ }^{\mathrm{a}}$, Peter A. Yaron ${ }^{\mathrm{a}}$ and John W.White*a \\ ${ }^{a}$ Research School of Chemistry, Australian National University, Canberra, Australia \\ ${ }^{*}$ Corresponding author atResearch School of Chemistry, Australian National University, Canberra 0200 \\ Australia.
}

\begin{abstract}
A strongly surface active, layered structure is formed at the air-water interface when an aqueous solution of fatted human serum albumin (F-HSA) is spread or mixed with a dilute sol of LS-30 silica nanoparticles at $25^{\circ} \mathrm{C}$. X-ray and neutron reflectivity show that the film is quickly established and is sensitive to the ionic strength of the sub-phase. The top layer is largely protein, the second contains silica and a third has diffuse protein. Optical circular dichroism in the mixtures shows that the silica changes the protein $\alpha$ helical content.
\end{abstract}

Keywords: human serum albumin, nanoparticle, structure, toxicology

\section{Introduction}

The interaction of proteins with nanoparticles is of current interest in nano-toxicology $[1,2,3]$, the "protein corona" hypothesis suggesting a mechanism for facilitated access of protein coated nanoparticles into human cells. We have previously shown, using $\beta$ casein - silica $[4,5]$, that protein- silica binding can be readily detected through induced surface activity using x-ray and neutron surface reflectivity - since the reflectivity from the sol sub-phase for both types of radiation is very weak.

This paper scopes some of the qualitative features of the same induced surface activity for silica-fatted human serum albumin (F-HSA) - where the protein is much more soluble than $\beta$ casein. The indication is that F-HSA, the most prolific protein component of blood, also forms a "corona" with silica and the resulting complex may be of eventual toxicological interest [6]. We expect a similar behaviour for other proteins in bodily fluids from the combined effects of electrostatic and van der Waals interactions. Here we show that both must be present, as well as possible specific interactions identified by Livage [7] between peptides and silica around neutral $\mathrm{pH}$.

\section{Materials and methods}

A $0.6 \mathrm{v} / \mathrm{v}$ percent sol of commercial LS-30 (Ludox) silica in milliQ water-based imidazole buffer $\mathrm{pH}$ 6.9 and lyophilized F-HSA (A8763) a defatted HSA (DF-HSA) (A3782), with purity greater than 99\%, from Sigma-Aldrich were used without any further purification. Both x-ray and neutron reflectivity were measured from this surface [8]and found, within experimental error, to be the same as a water surface. In the x-ray experiment a0.1mg of fatted human serum albumin in imidazole buffer was dropped evenly onto the sol solution with a micro-syringe and the interfacial reflectivity for $\mathrm{x}$-rays measured immediately and after approximately two hours, ten hours and twenty hours, with the surface enclosed to avoid evaporative effects. For the neutron scattering experiments at the SURF reflectometer (ISIS, Rutherford Appleton Laboratory, UK)the same procedure was followed for the protein alone and dropped on to $0.6 \% \mathrm{v} / \mathrm{v}$ LS-30 Ludox in imidazole buffer made up to be air contrasted matched (ACMW).

\section{Results}

\section{$3.1 X$-ray reflectivity}
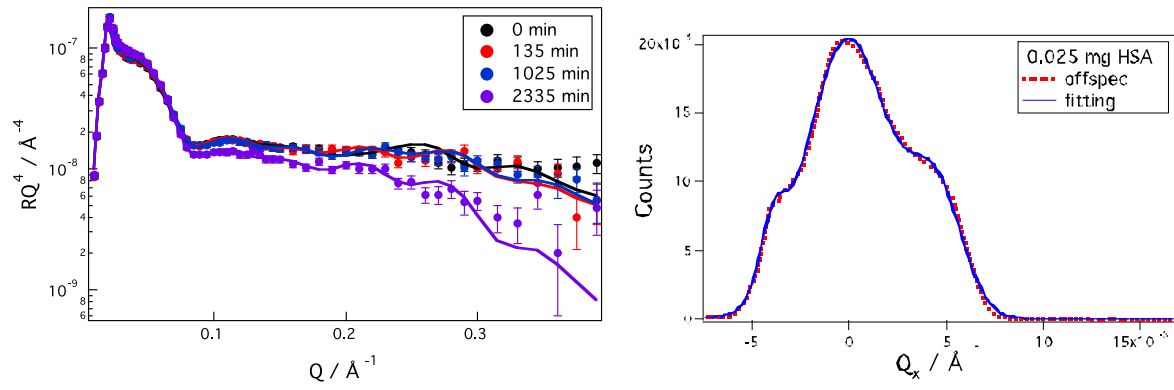
(a)

(b)

Figure 1 (a) X-ray reflectivity as RQ4 versus Q for $0.025 \mathrm{mg}$ of fatted human serum albumin spread on $0.6 \mathrm{v} / \mathrm{v} \% \mathrm{LS}-30$ silica as a function of time and (b) the off-specular scattering from the protein- silica sol after 2335 minutes at $25 \mathrm{C}$.

Figure 1 shows the x-ray reflectivity. The reflectivity has been multiplied by the fourth power of the momentum transfer (to minimize the free surface (Fresnel) component of the reflectivity and to emphasize the surface layer fringes). Three features are notable in the raw data - the fringe at $0.05 \AA^{-1}$, and another broad fringe at higher $\mathrm{Q}$ as well as a considerable increase in the roughness of the film upon standing for two days.

This roughness gives rise to "off-specular " scattering around the specular ridge. From the specular scattering, the parameters for the depth profile of the composite film are found. From the off-specular scattering, it is obvious that the surface roughness has some periodicity. As the films were not compressed during the time interval of observation, we attribute the in-plane periodicity to chemically induced stresses. From the position of the main peaks, ca $4 \times 10-4 \AA^{-1}$, the period of the fluctuations is of the order of 15 microns.

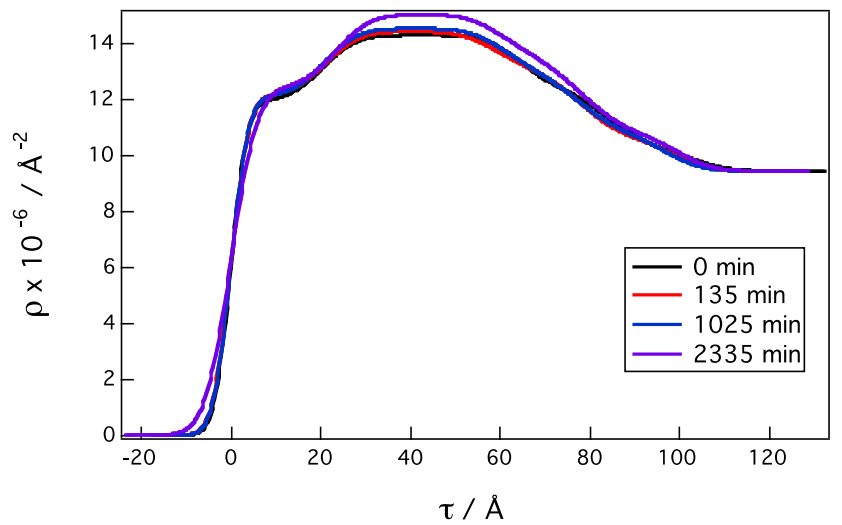

Figure 2Real space $\mathrm{x}$-ray scattering length density profiles from the air-water interface into the $0.6 \mathrm{v} / \mathrm{v} \%$ silica sol.

The specular reflectivity data were fitted using MOTOFIT [9] with initial constraints on both the protein and the nanoparticle scattering length density before refinement. Constraints on the roughness were also possible. Figure 2 shows the real space scattering length density distribution at each time of measurement. The distribution corresponds to that expected [4] for a mixture of weaker scattering protein and strongly scattering nanoparticle, with protein above and below the mixed nanoparticle protein region. The Figure shows the quick formation of the film and its stability with time.

Table 1 Fitting parameters used to produce the real space scattering length density profiles of Figure 2.

\begin{tabular}{llllll}
\hline & & $0 \mathrm{~min}$ & $135 \mathrm{~min}$ & $1025 \mathrm{~min}$ & $2335 \mathrm{~min}$ \\
\hline \multirow{3}{*}{ 1st layer } & Thickness & 20.3 & 21.0 & 20.8 & 23.5 \\
& SLD & 11.9 & 12.1 & 12.1 & 12.4 \\
& Roughness & 2.9 & 3.2 & 3.1 & 4.6 \\
\hline \multirow{3}{*}{ 2nd layer } & Thickness & 43.8 & 37.3 & 39.5 & 36.6 \\
& SLD & 14.3 & 14.4 & 14.5 & 15.0 \\
& Roughness & 6.0 & 6.0 & 6.0 & 6.0 \\
\hline \multirow{3}{*}{ 3rd layer } & Thickness & 20.0 & 19.4 & 17.3 & 18.2 \\
& SLD & 12.5 & 13.2 & 13.2 & 6.0 \\
\hline \multirow{3}{*}{ 4th layer } & Roughness & 6.0 & 6.0 & 6.0 & 21.7 \\
& Thickness & 19.7 & 21.4 & 20.1 & 10.7 \\
& SLD & 10.2 & 10.5 & 10.7 & 8.0 \\
\hline
\end{tabular}

The scattering length density of the top layer agrees well with that expected for almost pure protein $\left(12.2 \times 10^{-6} \AA^{-2}\right)$ and that for the middle layer is lower than pure silica - indicating a mixture of protein, silica and sub-phase water in this layer. 


\subsection{Neutron scattering experiments}

Neutron scattering experiments with air contrast matched sub-phase allow further definition of the relative positions and amounts of protein and nanoparticle in the two dimensional surface film.

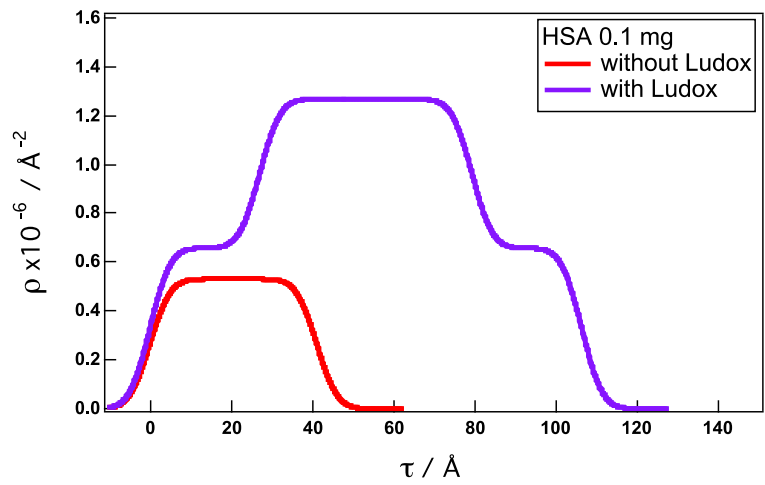

Figure 3 Neutron Scattering length density profiles from the air into the sub-phase for $0.1 \mathrm{mg}$ of F_HSA dropped onto buffer solution (red) and on to $0.6 \mathrm{v} / \mathrm{v} \%$ LS-30 buffered Ludox Silica (blue).

The neutron data for 0.1mgof the protein alone dropped on to ACMW imidazole buffer solution and on to $0.6 \% \mathrm{v} / \mathrm{v}$ LS-30 silica solin the same buffer(real space distributions after refinement) are shown in Figure 3. The protein film alone was measured first and, at the resolution available with the SURF spectrometer, has a distribution in accordance with published data [10]although the SLD of F-HSA in ACMW is lower than the expected value $\left(1.8 \times 10^{-6} \AA^{2}\right)$. This is consistent with the protein layer being strongly hydrated.

The SLD distribution from the protein nanoparticle combination, shows the growth in the density between $30 \AA$ to $60 \AA$ below the interfacial surface and its time dependence. Again, all SLD's are lower that those of the isolated materials and so we conclude that the surface film is gel-like. The fitted parameters are shown in Table 2.

Table 2 Fitting parameters for the protein only and the protein-silica film

\begin{tabular}{llll}
\hline & & Pure HSA & With Ludox \\
\hline \multirow{3}{*}{ 1st layer } & Thickness & 41.0 & 27.0 \\
& SLD & 0.53 & 0.66 \\
& Roughness & 4 & 4 \\
\hline \multirow{3}{*}{ 2nd layer } & Thickness & N/A & 52.2 \\
& SLD & N/A & 1.3 \\
& Roughness & N/A & 4 \\
\hline \multirow{3}{*}{3 rd layer } & Thickness & N/A & 27.00 \\
& SLD & N/A & 0.66 \\
& Roughness & N/A & 4 \\
\hline
\end{tabular}

Figure 4 shows the neutron reflectivity data for $0.1 \mathrm{mg}$ of F-HAS dropped on to the silica sol containing sodium chloride concentrations in the sub-phase between zero and 200mMolar. Again the three mixed layers are observed.

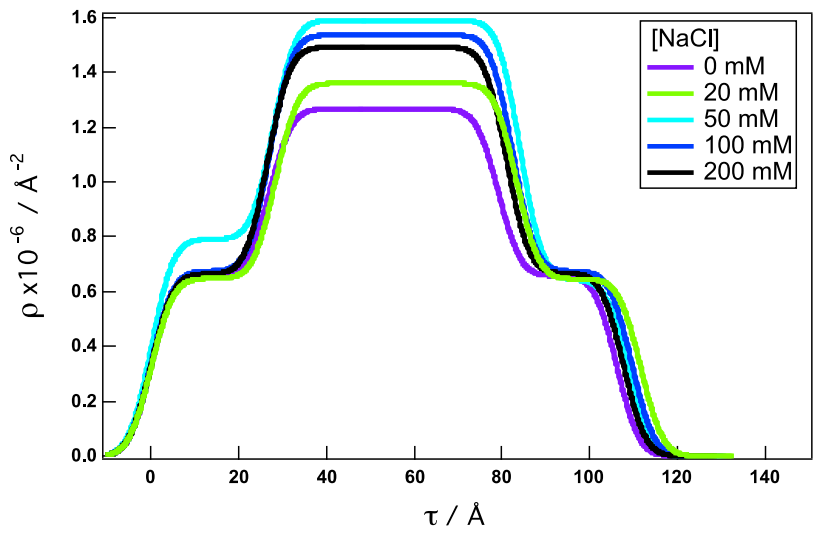


Figure 4Neutron Scattering length density profiles from the air into the sub-phase for $0.1 \mathrm{mg} / \mathrm{ml} \mathrm{F-HSA} \mathrm{buffer}$ solution with 0 to 200 milli Molar sodium chloride.

Table 3 . Parameters used to fit the neutron reflectivity from ACMW salt containing LS-30/ F-HSA

\begin{tabular}{lllllll}
\multicolumn{7}{c}{ mixtures } \\
\hline $\mathrm{NaCl}$ concentration & $0 \mathrm{mM}$ & $20 \mathrm{mM}$ & $50 \mathrm{mM}$ & $100 \mathrm{mM}$ & $200 \mathrm{mM}$ \\
\hline \multirow{2}{*}{$1^{\text {st }}$ layer } & Thickness & 27.0 & 28.4 & 27.8 & 27.1 & 26.6 \\
& SLD & 0.66 & 0.65 & 0.79 & 0.68 & 0.67 \\
& Roughness & 4 & 4 & 4 & 5 & 4 \\
\hline \multirow{2}{*}{$2^{\text {nd }}$ layer } & Thickness & 52.2 & 55.0 & 56.4 & 55.4 & 54.5 \\
& SLD & 1.27 & 1.36 & 1.59 & 4.54 & 1.49 \\
& Roughness & 4 & 4 & 4 & 27.1 & 4 \\
\multirow{2}{*}{$3^{\text {rd }}$ layer } & Thickness & 27.00 & 28.3 & 24.5 & 0.67 & 0.67 \\
& SLD & 0.66 & 0.65 & 0.64 & 4 & 4 \\
\hline
\end{tabular}

The data show significantly more silica in the middle layer with salt than without salt. The maximum amount occursat about $50 \mathrm{~m}$ molar salt. The sensitivity of the reflectivity indicates a weakened electrostatic component of the interaction and the likely importance of hydrophobic interactions near $\mathrm{pH} 7$.

\subsection{Interfacial structure}

\section{Discussion}

The high surface area $\left(215 \mathrm{~m}^{2} / \mathrm{gm}, \mathrm{pH} 8.2\right)$ of LS-30 Ludox makes it a highly reactive sol and a good candidate for exploring nanotoxicological properties. This particle was chosen because of its likely toxic properties and indeed there is evidence of this [6] as well as cautions in the manufacturer's specifications against irritant and related effects.

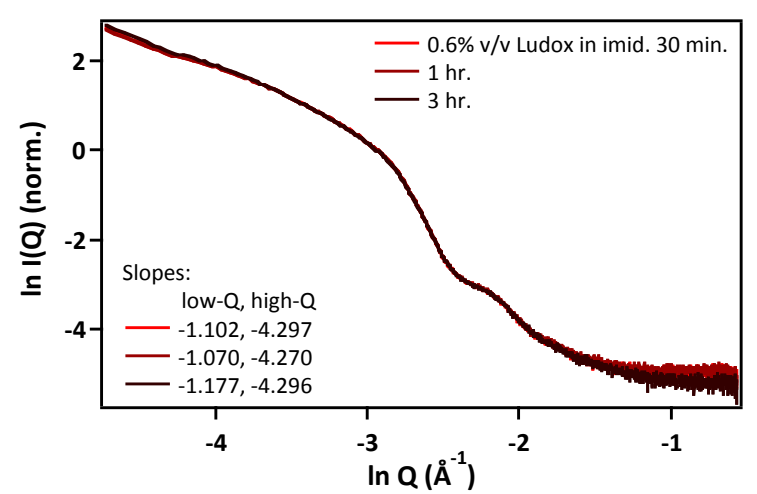

Figure 5 X-ray small angle scattering from freshly made $0.6 \% \mathrm{v} / \mathrm{v}$ LS-30 Ludox in standard phosphate buffer at $\mathrm{pH} 7$

For nanotoxicological comparisons, the nature of the silica was characterized by small angle $\mathrm{x}$-ray scattering. Although freshly diluted, the low Q slope in the small angle x-ray pattern of the $0.6 \mathrm{v} / \mathrm{v} \% \mathrm{pH} 7 \mathrm{sol}$ shown in Figure 5suggests some aggregation. No further changes occur over a three hour period. The fringe structure shows a polydispersity of about $20 \%$ and a mean particle radius of $41 \AA$ calculated from fitting the data of Figure 5 with a Sztuki model (11). This radius and the manufacturer's weight fraction give a consistent particle number of approx. $10^{18}$ particles per gram of the $30 \mathrm{w} / \mathrm{w} \%$ sol.

In all reflectivity experiments, a three layer structure was observed for the surface film - the middle layer, from its elevated scattering length density, containing silica. Only for the x-ray experiments, where the surface film was produced by dropping protein solution onto the silicate sol surface, were the scattering length densities of the top and bottom layers close to those expected for the x-ray scattering length densities of the protein in water. This suggests that this method of preparation produced more compact protein layers and, indeed, a different ration of silica to protein in the composite than for the pre-mixed solutions.

In the neutron measurements with air-contrast matched water, the protein layers had smaller scattering length densities than expected for protein-only layers and the middle layer never attained a scattering length density comparable to that of a silica-only structure. We thus conclude that all layers have some relatively large component of the aqueous medium and that again the silica content of the middle layer is nowhere near $100 \%$.

The results are thus similar to those found for the $\square$ casein-Ludox system and though compatible with the "corona" hypothesis, are not proof of it. The mean surface area of the LS-30 particle is $22 \times 10^{3} \AA^{2}$ and that of the F-HSA molecule - assumed ellipsoidal with minor and major axes $30 \times 50 \AA^{2}=1500 \AA^{2}$. Thus a monolayer of approximately 20 F-HSA molecules can be formed if that were the mechanism. Fewer contacts may arise 
because both the protein and the silica at $\mathrm{pH} 7$ are negatively charged with specific interactions and hydrophobic forces determining the attractive art of the potential to mediate the number adsorbed.

4.2 Protein secondary structure changes

The literature shows that protein secondary structure may be changed by adsorption, so UV circular dichroism measurements were made at $20^{\circ} \mathrm{C}$ on human serum albumin/LS-30 mixtures. A quartz cuvette with 1 $\mathrm{mm}$ path-length and nitrogen purged Chirascan spectrometer (Applied Photophysics, UK) was used between 190 and $320 \mathrm{~nm}$. The HSA concentration $(0.0625 \mathrm{mg} / \mathrm{ml})$ was chosen to keep the absorbance value lower than c.a. 2 at $190 \mathrm{~nm}$. The mean residue ellipticity $[\theta]\left(\mathrm{deg} \cdot \mathrm{cm}^{2} \cdot \mathrm{dmol}^{-1}\right)$ was calculated using the equation $[\theta]=\left(\theta_{o b s} / 10\right)(M W / n l c)$, where $\theta_{o b s}$ is the measured ellipticity (deg), $M W$ is the molecular weight of the protein, $n$ is the number of amino acid residues, $l$ is the optical path-length $(\mathrm{cm})$ and $c$ is the protein concentration $(\mathrm{mg} / \mathrm{ml})$.

The $\square \square$ helical content estimated using the CDPRO program [12] for solutions containing $0.03,0.3$ and $3 \mathrm{v} / \mathrm{v} \%$ LS-30 in standard phosphate buffer (to avoid imidazole absorption) The defatted form of the albumin (DF-HSA)was measured for comparison as the two molecules have very similar crystal structures $[13,14]$ but the fatted form contains up to six molecules of palmitic acid held in hydrophobic binding sites.
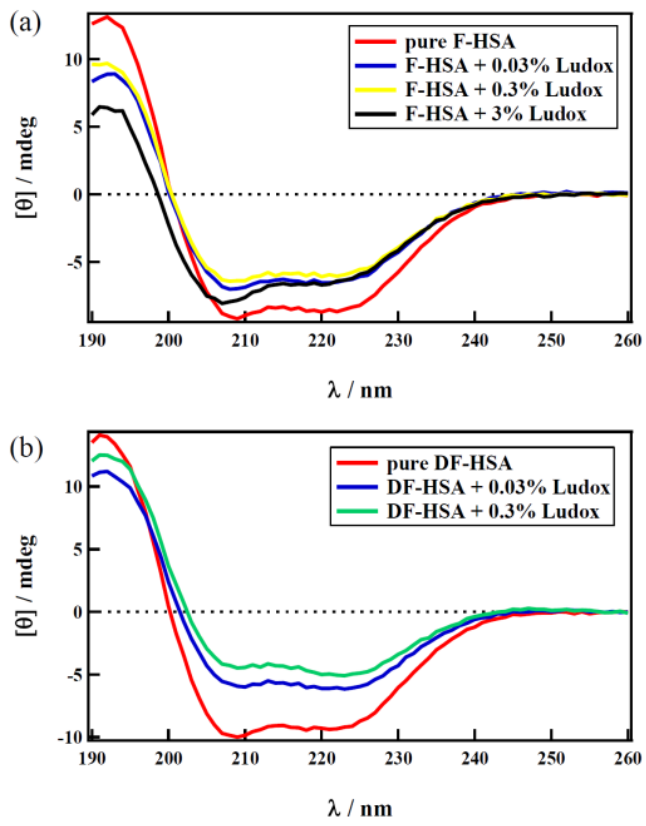

Figure6 CD spectra of protein-nanoparticle mixtures for (a) F-HSA and (b) DF-HSA.

In Figure 6 the two peaks at $208 \mathrm{~nm}$ and $222 \mathrm{~nm}$ are characteristic of the $\alpha$-helicesand appear for both pure fatted and pure defatted HSA. The CD signal changes as the concentration of nanoparticles is increased by a factor of 100. The $\alpha$-helical content of both F-HSA and DF-HSA decrease but for fatted human serum albumin the decrease is only a few percent. For DF-HSA the helix content falls from $54 \%$ to about $37 \%$. This resultis consistent with the denaturation results of Leggio et al.[15]that the fatty acids in F-HSA confer structural stability and hence resistance to nanoparticle-induced denaturation and the conformation changes of human hemoglobin seen on silica [16].

\section{Acknowledgements}

This work was financially supported by grants from the Australian Government's "Access to major overseas facilities" program.

\section{References}

[1] I. Lynch, T. Cedervall, M. Lundqvist, C. Cabaleiro-Lago, S. Linse, K.A. Dawson, The nanoparticle-protein complex as a biological entity; a complex fluids and surface science challenge for the $21^{\text {st }}$ century, Adv.Colloid Interface Sci.134 (2007) 167-174.

[2] M.P. Monopoli, C. Arberg, A. Salvati, K.A. Dawson, Biomolecular coronas provide the biological identity of nano-sized materials, Nat. Nanotechnol. 207 (2012) 779-786.

[3] D.A. Winkler, E. Mombelli, A. Pietroiusti, L. Tran, A. Worth, B. Fadeel, M.J. McCall, Applying quantitative structure-activity relationship approaches to nanotoxicology: Current status and future potential, Toxicol.(2012)http://dx.doi.org/10.1016/i.tox.2012.11.005.

[4] J.C. Ang, J-M. Lin, P.N. Yaron, J.W. White, Protein trapping of silica nanoparticles, Soft Matter 6 (2010) 383-390. 
[5] J.W. White, J-M. Lin, J.C. Ang, R.A. Campbell, V. Laux, M. Haertlein, G. Fragneto, Nanostructure of the "protein-nanoparticle corona" an indicator of toxicity? International Conference on Nanoscience and Nanotechnology (ICONN), 2010,doi/10.1109/ICONN.2010.6045250289-292.

[6] C.J.Leen,A.A. Thomassen,V.Rabolli,D. Lison,L.Gonzalez,M.Kirsch-Volders,D.Napierska, P.H.Hoet,C.E.A.Kirschhock,J.A Martens, Synthesisandcharacterization of stablemonodispersesilicananoparticlesolsforinvitrocytotoxicitytesting,Langmuir26 (2010)328-335.

[7] T. Coradin, O. Durupthy, J. Livage, Interactions of amino-containing peptides with sodium silicate and colloidal silica: a biomimetric approach of silicification,Langmuir18 (2002)2331-2336.

[8] A.S.Brown,S.A.Holt,P.M.Saville, J.W.White,Neutron and x-ray reflectometry - solid multilayers and crumpling films, Aust.J.Phys.50 (1997)391-405.

[9] A.Nelson, Co-refinement of multiple-contrast neutron/x-ray reflectivity data using MOTOFIT,J.Appl.Crystallogr.39 (2006)273-276.

[10] J.R. Lu, T.J. Su, J. Penfold, Adsorption of serum albumins at the air-water interface, Langmuir15 (1999) 6975-6983.

[11] M. Sztucki , T. Narayanan, G. Belina, A.Moussaïd, F. Pignon, H . Hoekstra Kinetic arrest and glass-glass transition in short-ranged attractive colloids, Physical Reviews E74(2006) 051504-12.

[12] J. Zhai, T.J. Wooster, S.V. Hoffmann, T.H. Lee, M.A. Augustin, M.I. Aguilar,Structural rearrangement of $\beta$-lactoglobulin at different oil-water interfaces and its effect on emulsion stability,Langmuir 27 (2011) 9227-36.

[13] S.Curry, H. Mandelkow, P. Brick, N. Franks,Crystal structure of human serum albumin complexed with fatty acid reveals an asymmetric distribution of binding sites, Nature Struct. Biol.5 (1998)827-835.

[14] A.A. Bhattacharya, T. Grune, S. Curry, Crystallographic analysis reveals common modes of binding of medium and long-chain fatty acids to human serum albumin, JMolecul. Biol.303 (2000) 721-732.

[15] C. Leggio, L. Galantini, P.V. Konarev, N.V. Pavel, Urea-induced denaturation process on defatted human serum albumin and in the presence of palmitic acid,J. Phys. Chem. B113 (2009) 12590-12602.

[16] A.Kondo,H.Fukuda, Effects of adsorption conditions on kinetics of protein adsorption and conformational changes at ultrafine silica particles, J.Colloid InterfaceSc.198(1998)34-41. 